\title{
Emergency Detection System using PDA based on Self-response Algorithm
}

\author{
Ah-young Jeon, Jun-mo Park, and Gyerok Jeon ${ }^{\mathrm{a}}$ \\ Department of Biomedical Engineering, College of Medicine, Pusan National University, \\ Pusan National University Hospital, Ami-dong 1-10, Seo-gu, Busan 602-739, Korea \\ Soo-young Ye \\ BK21 Medical Science Education Center, College of medicine, Pusan National University, \\ Pusan National University Hospital, Ami-dong 1-ga, Seo-gu, Busan 602-739, Korea \\ Jae-hyung Kim \\ School of Computer Aided Science, Inje University, \\ 607 Eobang-dong, Gimhae-si, Gyeongnam 621-749, Korea \\ ${ }^{a}$ E-mail: grieon@pusan.ac.kr
}

(Received November 8 2007, Accepted December 18 2007)

\begin{abstract}
The aged are faced with increasing risk for falls. The aged have more fragile bones than others. When falls occur, it is important to detect this emergency state because such events often lead to more serious illness or even death. A implementation of PDA system, for detection of emergency situation, was developed using 3-axis accelerometer in this paper as follows. The signals were acquired from the 3-axis accelerometer, and then transmitted to the PDA through a Bluetooth module. This system can classify human activity, and also detect an emergency state like falls. When the fall occurs, the system generates the alarm on the PDA. If a subject does not respond to the alarm, the system determines whether the current situation is an emergency state or not, and then sends some information to the emergency center in the case of an urgent situation. Three different studies were conducted on 12 experimental subjects, with results indicating a good accuracy. The first study was performed to detect the posture change of human daily activity. The second study was performed to detect the correct direction of fall. The third study was conducted to check the classification of the daily physical activity. Each test lasted at least $1 \mathrm{~min}$. in the third study. The output of the acceleration signal was compared and evaluated by changing various postures after attaching a 3-axis accelerometer module on the chest. The newly developed system has some important features such as portability, convenience and low cost. One of the main advantages of this system is that it is available at home healthcare environment. Another important feature lies in its low cost of manufacture. The implemented system can detect the fall accurately, so it will be widely used in emergency situations.
\end{abstract}

Keywords : Emergency, 3-axis accelerometer, Self-response, PDA system

\section{INTRODUCTION}

Since the birth rate has decreased, whereas the average span of life has increased, people who have reached age 65 or older are the fastest growing population of the world. So, we are currently faced with a wide range of problems which are related to the aging society, including the emerging needs of home-visit nursing and ubiquitous healthcare. In particular, the aged are at an increased risk for falls. When a fall occurs, it is important to detect the situation promptly because such accidents often lead to more serious illness or even death. The early detection of an emergency- like falls is essential to rescue the injured from danger and obtain aid as fast as possible.

So, monitoring and classifying human movement is important. In the past, the ambulatory measurement of the physical activity was based on various motion sensors, such as pedometers and act meters. Recently the accelerometer has been employed to facilitate such long- 
term monitoring of human motion by using the wearable sensor unit [1,2]. And, many systems utilized the multiple accelerometer units which is placed at various sites of the body to assist their detection of activities, such as walking, ascending and descending stairs, and cycling[35]. In another research, a kinetic sensor, which is composed of one miniature piezoelectric gyroscope and two miniature accelerometers, was used to analyze human motion[6,7]. Furthermore, new systems were developed to identify the static and dynamic activity using 3-axis accelerometer[8-10]. However, these methods are somewhat cumbersome because they needed two or more different sites of sensor attachment to the body and cable connection, which reduced their applicability for long-term monitoring physical activity and interfering activities.

The PDA system was developed in this research using 3-axis accelerometer, which could classify human activity and detect an emergency state more conveniently. This system can easily detect the posture change, fall, and daily physical activity. The system can generate the alarm on the PDA (Acode-300, Chipsen, Co. Korea), when an urgent situation like the fall is detected. If the subjects do not respond to the alarm, the system will determine that the current situation is an emergency state, and then informs the emergency center.

\section{METHODS}

\subsection{Instrumentation}

The proposed system can acquire the $\mathrm{x}$-axis (left $(+)-$ right (-)), y-axis (up (+) - down (-)), z-axis (rear $(+)-$ front $(-)$ ) signals from the 3-axis accelerator (MMA $7260 \mathrm{Q}$ Free scale Co. USA), monitor the signals via PDA, and detect an emergency state like falls.

Figure 1 shows the schematic diagram of the PDA system for detecting an emergency state. The different 3axis signals were generated as a motion of the subject in a specifie situation such as walking, lying down, and so on. The whole block diagram of the system was shown in Fig. 2.
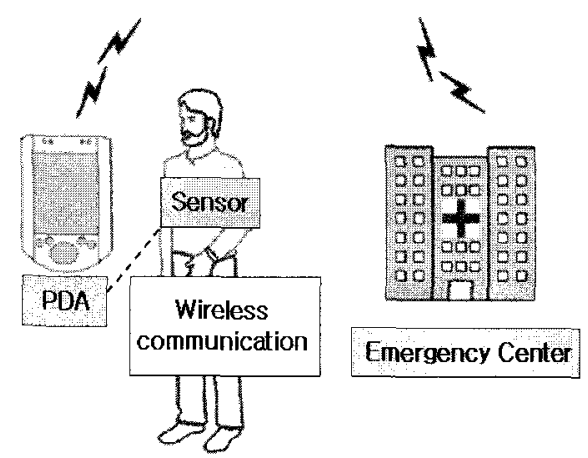

Fig. 1. Schematic of PDA system for detecting emergency state.

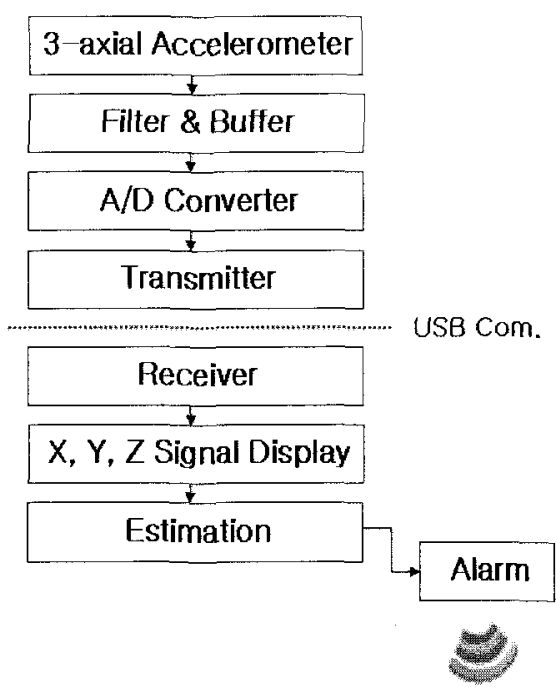

Fig. 2. Block diagram of the system.

The output signal $(\mathrm{X}, \mathrm{Y}, \mathrm{Z})$ of 3-axis accelerometer transmits to three channels of the DAQ board. These signals transmit to PDA via Bluetooth module. The acquired signals were analyzed via LabVIEW software (LabVIEW 7.1.1, N.I. Co., U. S. A.).

\subsection{Experimental protocol}

The signals were acquired using the 3-axial accelerometer which was attached on the chest of the subject to detect signals according to the posture change. The experimental protocols of the posture change and fall, and daily physical activity are represented respectively in Tables I, II, III.

Table 1. Experiment $1:$ Description of activities performed by each subject to analyze human activity.

\begin{tabular}{|c|c|c|}
\hline Task & Description & $\begin{array}{c}\text { Duration } \\
\text { (s) }\end{array}$ \\
\hline Sit-to-stand & $\begin{array}{l}\text { From an initially seated } \\
\text { position, the subject stands up } \\
\text { and remains standing }\end{array}$ & 10 \\
\hline Stand-to-sit & $\begin{array}{l}\text { From an initially stranding } \\
\text { position, the subject sits down } \\
\text { and remains seated }\end{array}$ & 10 \\
\hline Sit-to-lying & $\begin{array}{l}\text { Initially sitting up on the } \\
\text { mattress, the subject lies down } \\
\text { on their back and remains in } \\
\text { this position }\end{array}$ & 10 \\
\hline Lying-to-sit & $\begin{array}{l}\text { Initially lying on the their back } \\
\text { on the mattress, the subject } \\
\text { sits up and remains in this } \\
\text { position }\end{array}$ & 10 \\
\hline Stand-to-walking & $\begin{array}{l}\text { From an initially standing } \\
\text { position, the subject walks and } \\
\text { remains in this position. }\end{array}$ & 10 \\
\hline $\begin{array}{l}\text { Lying } \\
\text { (back-right-back } \\
\text {-left-back) }\end{array}$ & $\begin{array}{l}\text { From an initially lying } \\
\text { backward (5 s), rightward } \\
(5 s) \text {, backward (5s), leftward } \\
(5 s) \text {, and backward }\end{array}$ & 25 \\
\hline
\end{tabular}


Table 2. Experiment 2 : Description of activities performed by each subject to detect falls.

\begin{tabular}{clc}
\hline Task & \multicolumn{1}{c}{ Description } & $\begin{array}{c}\text { Duration } \\
(\mathrm{s})\end{array}$ \\
\hline Frontal fall & $\begin{array}{l}\text { Initially standing up, the subject falls } \\
\text { frontward onto the mattress } \\
\text { Initially standing up, the subject falls } \\
\text { backward onto the mattress }\end{array}$ & 10 \\
Left fall & $\begin{array}{l}\text { Initially standing up, the subject falls } \\
\text { leftward onto the mattress } \\
\text { Right fall }\end{array}$ & 10 \\
\hline
\end{tabular}

Table 3. Experiment 3 : Description of activities performed by each subject to classify the daily physical activity.

\begin{tabular}{lll}
\multicolumn{1}{c}{ Task } & \multicolumn{1}{c}{ Description } & $\begin{array}{c}\text { Duration } \\
\text { (s) }\end{array}$ \\
\hline $\begin{array}{l}\text { Stand-walking- } \\
\text { stand-sit-lying-sit- } \\
\text { stand }\end{array}$ & $\begin{array}{l}\text { Initially standing up, the } \\
\text { subject is walking, } \\
\text { standing, sitting, lying, } \\
\text { sitting and standing. }\end{array}$ \\
Stand-walking- & $\begin{array}{l}\text { Initially standing up, the } \\
\text { stand-sit-lying-sit- } \\
\text { stand } \\
\text {-fall }\end{array}$ & $\begin{array}{l}\text { standing, sitting, lying, } \\
\text { sitting, standing, and } \\
\text { falling forward onto the } \\
\text { mattress }\end{array}$ \\
\hline \hline
\end{tabular}

Three different studies which are posture change, fall, daily physical activity, were conducted on the 12 experimental subjects. The first study was conducted to check the correct analysis of the posture change. The experimentation protocol of posture change was represented in Table 1.

The second study was conducted to check the correct detection of fall according to the direction. The subjects performed 4 different tests including frontal fall, back fall, left fall and right fall as described in Table 2.

The third study was conducted to check the classification of daily physical activity. Each test lasted at least $1 \mathrm{~min}$. with respect to the task in this experiment. Activities performed by subjects were described in Table III.

\subsection{Algorithm of classifying the human activity}

To distinguish the experimental subject's activity, that is posture change, fall, and daily physical activity, the classifying algorithm of the human activity was proposed in this study. Figure 3 shows the block diagram of activity classification.

A suitable parameter for detection of the subject's behavior aspect, discussed in previous studies[1] is normalized Signal Magnitude Area (SMA).

$$
S M A=\frac{1}{t}\left(\int_{0}^{t}|x(t)| d t+\int_{0}^{t}|y(t)| d t+\int_{0}^{t}|x(t)| d t\right)
$$

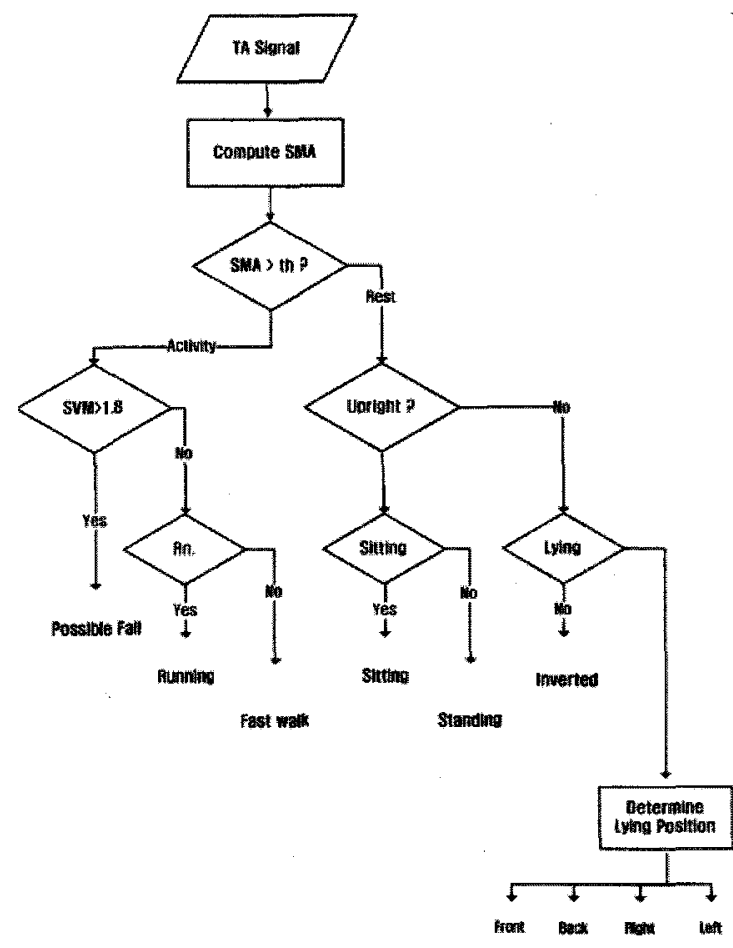

Fig. 3. Classifying algorithm of the human activity.

The SMA defined in equation (1) is used as a basis for identifying the subject's behavior aspect, which is running, fast walking, sitting, standing, and lying.

Where $x(t), y(t)$, and $z(t)$ refer to the acquired sample signals according to posture change from the $\mathrm{x}, \mathrm{y}$, and $\mathrm{z}$ axis accelerometer, respectively. Calculation of this parameter is performed by summing each sample's value progressively over a 1 second interval. An appropriate threshold value was determined via testing-for an SMA value, in which activity was deemed to have occurred, and values below the threshold is considered in the resting state.

\subsection{Algorithm of detecting the falls}

Falls have occurred if at least two consecutive peaks in the signal magnitude vector (SVM) above a defined threshold are recorded.

$$
S V M=\sqrt{x_{i}^{2}+y_{i}^{2}+z_{i}^{2}}
$$

SVM, defined in equation (2), essentially provides a measurement of the degree of movement intensity, as derived from the TA (output of the 3-axis accelerometer) output signal. The fall detection technique and the associated threshold are described in reference to the other research[1]. The threshold was determined by considering accelerations in SVM and in $\mathrm{x}, \mathrm{y}$, and $\mathrm{z}$-axis, whereas falls and stumbles were simulated. A review of these test results has identified the most suitable parameter to be SVM at a threshold of $1.8 \mathrm{~g}$. 


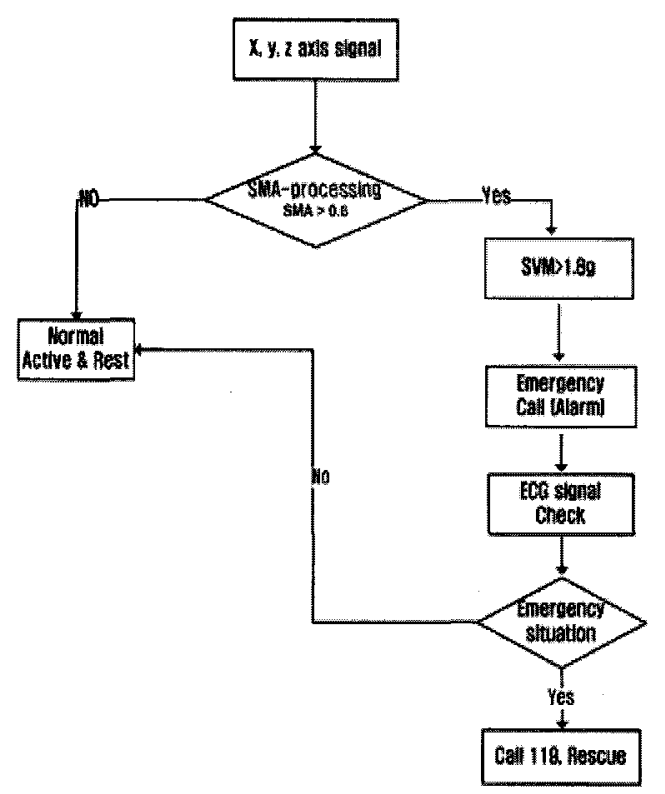

Fig. 4. Algorithm of detecting the falls.

The system generates the alarm on the PDA, when the fall is detected. If a subject does not respond to the alarm, the system determines whether the current situation is an emergency state or not, and sends some information to the emergency center in the case of an emergency as shown in Fig. 4.

\section{RESULTS}

The experimental subjects performed various tests using 3-axis accelerometer attached on the chest. Figure 5 shows the variation aspect of the acquired 3-asix accelerometer signals and SMA and SVM parameters according to the variation of walking speed, that is, slow walking (S.W), normal walking (N.W), fast walking (F.W), and fall down forward (F). Acquired data from each axis was normalized, with the assumption that $\mathrm{z}$ axis is always under the gravitational acceleration $1 \mathrm{~g}$ as the standard of signal magnitude.

As shown in the Fig. 5, the z-axis signal was indicated more closely related to the walking than $\mathrm{x}$-axis and $\mathrm{y}$ axis signal. The 3-axis signal, SMA and SVM increased according to the increase of the walking speed. And, the activity state could not be determined by analyzing the SMA and SVM.

Because the direction of fall down is front, the amplitude of the $\mathrm{x}$-axis and $\mathrm{z}$-axis signal were observed considerably higher than the experimental subject fall down. And SMA and SVM were observed also higher in this case, than in the walking state.

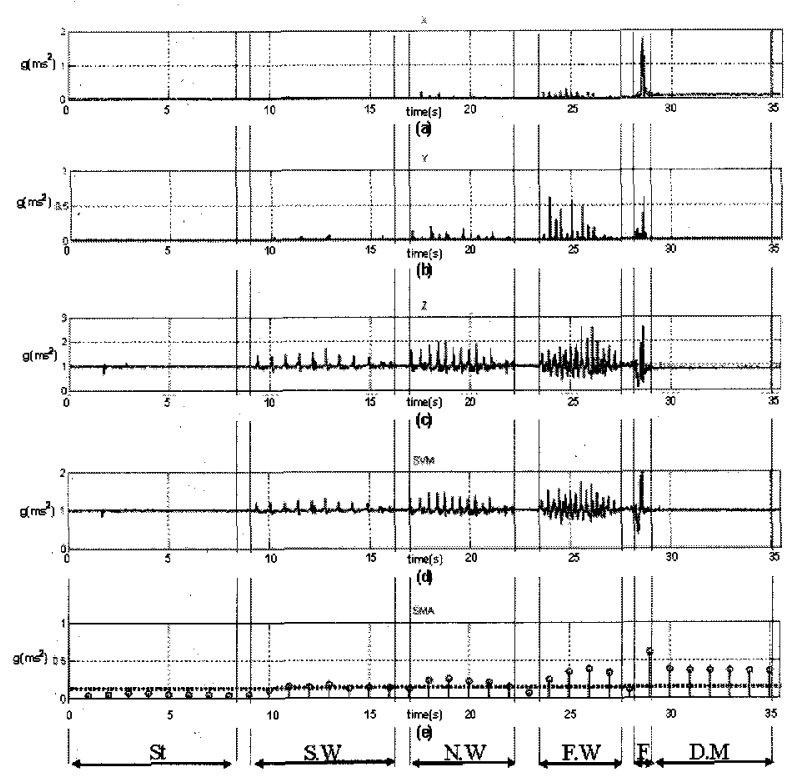

Fig. 5. Variation aspect of 3-axis accelerometer signal and SMA and SVM parameters after normalized process according to walking speed change and fall down: (a) $\mathrm{x}$ axis signal, (b) y-axis signal, (c) z-axis signal, (d) SVM, and (e) SMA.

Table 4. The mean and standard deviation of SMA and SVM according to walking speed change.

\begin{tabular}{cccccc}
\hline \multirow{2}{*}{ Pattern } & \multicolumn{2}{c}{ SMA (g) } & \multicolumn{2}{c}{ SVM (g) } \\
\cline { 2 - 6 } & & Mean & SD. & Mean & SD. \\
\hline \hline Slow & Woman & 0.15 & 0.05 & 1.15 & 0.05 \\
\cline { 2 - 6 } walking & Man & 0.19 & 0.03 & 1.21 & 0.08 \\
\hline Normal & Woman & 0.21 & 0.06 & 1.25 & 0.12 \\
walking & Man & 0.23 & 0.04 & 1.42 & 0.10 \\
\hline Fast & Woman & 0.35 & 0.05 & 1.60 & 0.21 \\
\cline { 2 - 6 } walking & Man & 0.37 & 0.04 & 1.92 & 0.19 \\
\hline Runnin & Woman & 0.51 & 0.05 & 2.05 & 0.26 \\
\cline { 2 - 6 } g & Man & 0.56 & 0.06 & 2.14 & 0.21 \\
\hline \multirow{2}{*}{ Fall } & Woman & 0.60 & 0.10 & 2.31 & 0.26 \\
\cline { 2 - 6 } & Man & 0.65 & 0.15 & 2.65 & 0.31 \\
\hline
\end{tabular}

The mean and standard deviation of SMA and SVM according to the variation of walking speed and fall was shown in Table IV.

The classified by activity pattern were not identified using the value of SMA and SVM in Table IV. But, SMA and SVM were distinguished, when the experiment subjects fell down. The value of SMA is approximately 0.6 , and the value of SVM is approximately $2.31 \sim 2.61$.

The accuracy of correctly classifying the activity in this system is shown in Table V. Experiment I and II was conducted three times on each subject, and Experiment III was conducted two times on each subject. 
Table 5. The mean and standard deviation of SMA and SVM according to walking speed change.

\begin{tabular}{|c|c|c|c|c|c|}
\hline $\begin{array}{l}\text { Experi } \\
\text { ment }\end{array}$ & Task & $\begin{array}{l}\text { Total } \\
\text { Test }\end{array}$ & $\begin{array}{l}\text { Overall } \\
\text { Correct }\end{array}$ & $\begin{array}{l}\text { Overall } \\
\text { Incorrect }\end{array}$ & $\begin{array}{c}\text { Accuracy } \\
(\%)\end{array}$ \\
\hline \multirow{6}{*}{1} & Sit-to-stand & 36 & 34 & 2 & 94.44 \\
\hline & Stand-to-sit & 36 & 35 & 1 & 97.22 \\
\hline & Sit-to-lying & 36 & 36 & 0 & 100 \\
\hline & Lying-to-sit & 36 & 36 & 0 & 100 \\
\hline & Stand-to-walking & 36 & 35 & 1 & 97.22 \\
\hline & $\begin{array}{l}\text { Lying(back-right- } \\
\text { back-left-back) }\end{array}$ & 36 & 29 & 7 & 80.55 \\
\hline \multirow{4}{*}{ II } & Frontal fall & 36 & 36 & 0 & 100 \\
\hline & Back fall & 36 & 36 & 0 & 100 \\
\hline & Left fall & 36 & 32 & 4 & 88.88 \\
\hline & Right fall & 36 & 35 & 1 & 97.22 \\
\hline \multirow{2}{*}{ III } & $\begin{array}{l}\text { Stand-walking- } \\
\text { stand-sit-lying-sit- } \\
\text { stand }\end{array}$ & 24 & 21 & 3 & 87.5 \\
\hline & $\begin{array}{l}\text { Stand-walking- } \\
\text { stand-sit-lying-sit- } \\
\text { stand-fall }\end{array}$ & 24 & 23 & 1 & 95.83 \\
\hline
\end{tabular}

Viewing the results as a whole, the accuracy of experiment I to analysis activity was $94.9 \%, 96.52 \%$ on the experiment II to detect falls, $91.665 \%$ on experiment III to classify the daily physical activity.

As above, the accuracy of left fall was $88.88 \%$ on experiment II to detect the fall. The reason for this is supposed that the whole of the subjects are right handed, so they're conscious of the experiment. Even though there was some error, the overall result of the study was considerably reliable.

\section{DISCUSSION AND CONCLUSION}

The PDA system was developed using the 3-axis accelerometer. This system could classify human activity, and also detect an emergency like a fall. The system generates the alarm on the PDA, when the fall is detected. If a subject does not respond to the alarm, the system determines whether the current situation is an emergency state or not, and then sends some information to the emergency center in the case of emergency.

This system has some important features such as portability, convenience and low cost which the previous systems did not meet.

However, one of the main advantages of this system is that it is available at home visit nursing and home healthcare environment. Most of the previous devices checked the wearer's conditions indoors. Even though there are a few outdoor devices, they are not only cost efficient but also utility efficient. It is certainly true that sometimes, many of the previous devices are inconvenient and complex to handle. Also, it is possible not to deal with emergency situation properly.
The device developed in this work is so small that it is easy to carry when the wearers go anywhere. The wearer just wears the device on the chest. When the wearer suddenly falls down due to any situation like slippery roads or disease, the device can detect and help obtain quick medical treatment through sending some information to the emergency center. It can provide prompt service for people in an emergency situation. It is especially beneficial when the wearer, such as the aged fall down where no one is around him or her. As soon as emergency center gets the information from the PDA, the center can identify where the aged fell down through signals of the device. The center can call an ambulance and send people who can provide help. It is certain that this device is very useful when the accident like a fall occurs.

Another important feature of our device is that it is very simple. In fact, business for the aged is expected to grow substantially. Therefore, many devices for the aged to cope with an emergency state have been invented in recent years. Many of them introduce high IT and BT technology which is costly. Therefore, people who need to use the devices are limited. But, the newly developed system has attempted to overcome these drawbacks by adopting the useful algorithm of detecting the emergency state like falls.

Moreover, the result of the test was considerably reliable. The developed system in this work can detect the fall accurately, so it can be widely used in ubiquitous health care and emergency monitoring.

\section{ACKNOWLEDGMENT}

This study was supported by a grant of the Korea Health 21 R\&D Project, Ministry of Health \& Welfare, Republic of Korea(Grant No:A040032).

\section{REFERENCES}

[1] C. V. Bouten, K. T. Koekkoek, M. Verduin, R. Kodde, and J. D. Janssen, "A triaxial accelerometer and portable data processing unit for the assessment of daily physical activity", IEEE Trans. Biomed. Eng., Vol. 44, No. 3, p. 136, 1997.

[2] M. J. Mathie, A. C. F. Coster, N. H. Lovell, and B. G. Celler, "A pilot study of long term monitoring of human movements in the home using accelerometry", J. Telemed.Telecare, Vol. 10, p. 144, 2004.

[3] J. Fahrenberg, F. Foerster, M. Smeja, and W. Muller, "Assessment of posture and motion by multichannel piezoresistive accelerometer recordings", Psychophysiol, Vol. 34, p. 607, 1997. 
[4] F. Foerster and J. Fahrenberg, "Mtion pattern and posture: correctly assessed by calibrated accelerometers", Behav. Res. Meth. Instrum. Comput., Vol. 32, p. 450, 2000.

[5] P. H. Veltink, H. B. Bussmann, W. de Vries, W. L. Martens, and R. C. van Lummel, "Detection of static and dynamic activities using uniaxial accelerometers", IEEE Trans. Rehabil. Eng., Vol. 4, p. $375,1996$.

[6] Dean M. Karantonis, Michael R. Narayanan, Merryn Mathie, Nigel H. Lovell, and Branko G. Celler, "Implementation of a real-time human movement classifier using a triaxial accelerometer for ambulatory monitoring", IEEE Trans. Info. Tech. Biomed., Vol. 10, No. 1, p. 156, 2006.

[7] B. Najafi, K. Aminian, F. Loew, Y. Blanc, and P.
Robert, "Falling risk evaluation in elderly using miniature gyroscope", in proc. Microtech. in Med. and Bio., 1st Annu. Int. Conf., p. 562, 2000.

[8] M. P. Wachowiak, G. S. Rash, P. M. Guesada, and A. H. Desoky, "Wavelet-based noise removal for biomechanical signals : A comparativie study", IEEE Trans. Biomed. Eng., Vol. 47, p. 360, 2000.

[9] M. Sekine, T. Tamura, M. Ogawa, T. Togawa, and Y. Fukui, "Classification of acceleration waveform in a continuous walking record", Proc. 20th Annu. Int. Conf. IEEE Engineering in Mdicine and Biology Soc., Vol. 20, No. 3, p. 1523, 1998.

[10] C. H. Park, K. S. Lee, J. B. Kim, and, J. H. Kim, "The moving photocarrier grating (MPG) technique for the transport properties of $a-S e$ : As films", Trans. EEM, Vol. 6, No. 6, p. 280, 2005. 